\title{
TITLE:
}

\section{Ionic excitation in dense, two- component plasmas: Effect of energy level shifts}

\author{
$\operatorname{AUTHOR}(\mathrm{S})$ :
}

Kitamura, $\mathrm{H}$

\section{CITATION:}

Kitamura, H. Ionic excitation in dense, two-component plasmas: Effect of energy level shifts. PHYSICS OF PLASMAS 2004, 11(2): 771-779

\section{ISSUE DATE:}

2004-02

URL:

http://hdl.handle.net/2433/50050

\section{RIGHT:}

Copyright 2004 American Institute of Physics. This article may be downloaded for personal use only. Any other use requires prior permission of the author and the American Institute of Physics. 


\title{
Ionic excitation in dense, two-component plasmas: Effect of energy level shifts
}

\author{
Hikaru Kitamura ${ }^{\text {a) }}$ \\ Department of Physics, Kyoto University, Sakyo-ku, Kyoto 606-8502, Japan
}

(Received 11 September 2003; accepted 28 October 2003)

Collisional excitation rates for an ion immersed in dense electron-ion two-component plasmas are formulated by taking into account shifts of ionic energy levels due to static and dynamic plasma perturbations. The theory is based on the equations of motion for density matrices combined with the statistical theory of plasma density fluctuations. Through separation of the time scales associated with electron- and ion-density fluctuations, the electron-induced excitation rates are derived, where energy level shifts arising from quasistatic electric microfields by screened plasma ions as well as those arising from time-averaged spherical plasma polarization are considered. As a numerical example, the transition rates among the $2 s$ and $2 p$ fine-structure levels of a $\mathrm{Ne}^{9+}$ ion in dense hydrogen plasmas are calculated. It is demonstrated that, at sufficiently high plasma densities, the ion microfields cause Stark splittings and affect the excitation rates significantly through modifications of the generalized oscillator strengths. (C) 2004 American Institute of Physics.

[DOI: $10.1063 / 1.1637344]$

\section{INTRODUCTION}

In the previous paper, ${ }^{1}$ we have studied collisional excitation of a highly charged ion embedded in hot dense plasmas relevant to the inertial-confinement-fusion research and experiments using high-power lasers. The theory is based on the density matrix formalism, and describes time evolutions of ionic level populations in the presence of plasma density fluctuations. When the transition is so weak that first-order perturbation theory is valid, the theory reduces to the stochastic perturbation approach ${ }^{2-5}$ based on the Fermi's Golden Rule: the excitation rate is expressed in terms of Bethe's generalized oscillator strengths ${ }^{6}$ and plasma density correlation functions (dynamic structure factors ${ }^{7}$ ). At high plasma densities, it has been shown ${ }^{1}$ that the contribution from low-frequency ion density fluctuations causes strong coherent excitations which cannot be treated by the perturbation theories.

In dense plasma environment, bound states of the target ion are expected to be modified substantially. The main purpose of this paper is to consider the effect of ionic energy level shifts on the excitation rates, which was completely neglected in our previous paper. ${ }^{1}$ Elucidation of energy level shifts in dense electron-ion two-component plasmas (TCPs) offers a difficult problem, because dynamical motions of electrons and ions have significantly different time scales and also they are correlated with each other via Coulomb interaction. When viewed in a sufficiently long time scale, the Coulomb field of the target ion induces spherical polarization of the surrounding plasma particles, which in turn produces the shift of bound-state energies. Such plasma polarization shifts were studied by solving the Schrödinger equations for bound electrons in static model potentials. ${ }^{5,8-10}$

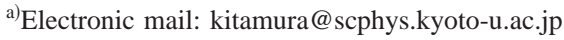

As a different origin of the energy level shift, we should also consider the slowly moving plasma ions that produce nonspherical, quasistatic electric microfields at the target ion within the time scale of the inelastic collision. ${ }^{11-14}$ Such a description may be applicable to electron-induced excitations, because the transitions are caused mainly by rapidly fluctuating electrons and the electric microfield by slowly moving ions can be regarded as quasistatic during the transition. ${ }^{13}$ As a result, the Stark splitting destroys the orientational degeneracies and breaks the sublevels into several closely spaced atomic states ${ }^{15}$ moreover, the Stark mixing modifies the generalized oscillator strengths. The importance of such ion microfield effect was demonstrated by Perrot ${ }^{11}$ who studied the influence of the Stark effect on the electronimpact excitation cross sections. Murillo ${ }^{13}$ likewise proposed to incorporate the Stark effect into the stochastic perturbation formula to study the electron-impact ionization processes in dense plasmas.

In this paper, we derive a formal expression for the ionic excitation rates in dense TCP, where the transitions and energy level shifts are treated on an equal footing on the basis of the density matrix formalism developed in our previous paper. ${ }^{1}$ The resultant formula takes a rather complicated form, but becomes tractable for electron-induced excitation where the transitions due to rapidly fluctuating electrons and energy level shifts due to slowly fluctuating ions can be treated separately. Numerical examples are given for the $n$ $=2$ fine-structure transitions ( $n$ : principle quantum number) of a $\mathrm{Ne}^{9+}$ ion in a hydrogen plasma.

\section{EXCITATION RATE FORMULA}

\section{A. General formulation}

We consider a hydrogen-like target ion with nuclear charge $Z e$, immersed in a classical two-component plasma 
consisting of electrons (charge number $Z_{e}=-1$, mass $m_{e}$, number density $n_{e}$ ) and ions (charge number $Z_{\mathrm{i}}$, mass $m_{i}$, number density $n_{i}=n_{e} / Z_{\mathrm{i}}$ ) all at temperature $T$. The Hamiltonian of the target ion in the time-dependent plasma field is written as

$$
H(t)=-\frac{\hbar^{2}}{2 m_{e}} \nabla^{2}-\frac{Z e^{2}}{r}+\sum_{\sigma=e, i} \sum_{j=1}^{N_{\sigma}} \frac{Z Z_{\sigma} e^{2}}{r_{j}^{(\sigma)}(t)}+V(\boldsymbol{r}, t) .
$$

Here, the position of the nucleus in the target ion is fixed at the origin, and $\mathbf{r}$ denotes the position of its bound electron; $\mathbf{r}_{j}^{(\sigma)}(t)$ is the position of the $j$ th plasma particle of species $\sigma$ at time $t$, and $N_{\sigma}=n_{\sigma} \Omega$, with $\Omega$ being the volume of the plasma. The interaction between the bound electron and all the plasma particles is expressed as

$$
\begin{aligned}
V(\boldsymbol{r}, t) & =\sum_{\sigma=e, i} \sum_{j=1}^{N_{\sigma}} \frac{-Z_{\sigma} e^{2}}{\left|\boldsymbol{r}-\boldsymbol{r}_{j}^{(\sigma)}(t)\right|} \\
& =\sum_{\sigma=e, i} \frac{1}{\Omega} \sum_{\boldsymbol{k}}\left(-Z_{\sigma}\right) v(k) \exp (i \boldsymbol{k} \cdot \boldsymbol{r}) \rho_{\sigma}(\boldsymbol{k}, t),
\end{aligned}
$$

where $v(k) \equiv 4 \pi e^{2} / k^{2}$ represents the Fourier transform of the Coulomb potential, and $\boldsymbol{\rho}_{\sigma}(\boldsymbol{k}, t)=\Sigma_{j=1}^{N_{\sigma}} \exp [-i \boldsymbol{k}$ $\left.\cdot \boldsymbol{r}_{j}^{(\sigma)}(t)\right]$ is the density-fluctuation operator for the plasma. ${ }^{7}$

We now develop a dynamic equation describing the excitation of the target ion from initial state $|1\rangle$ to final state $|2\rangle$. Following Ref. 1, we introduce the density matrix $\rho$ $=\left\{\rho_{m n}\right\}(m, n=1,2)$ for a two-level atom, which obeys the equation of motion, $\mathrm{d} \rho(t) / \mathrm{d} t=(i / \hbar)[\rho(t) H(t)-H(t) \rho(t)]$. The population of state $|n\rangle$ at time $t$ is then given by $\rho_{n n}(t)$. Since the ion is in state $|1\rangle$ at $t=0$, the equation of motion is integrated with the initial condition, $\rho_{11}(0)=1$ and $\rho_{22}(0)$ $=\rho_{12}(0)=\rho_{21}(0)=0$, to yield

$$
\begin{aligned}
\rho_{22}(t)= & \frac{i}{\hbar} \int_{0}^{t} \mathrm{~d} t_{1} \rho_{21}\left(t_{1}\right) V_{12}\left(t_{1}\right)+\mathrm{cc}, \\
\rho_{21}(t)= & \frac{i}{\hbar} \int_{0}^{t} \mathrm{~d} t_{2} V_{21}\left(t_{2}\right)\left[\rho_{22}\left(t_{2}\right)-\rho_{11}\left(t_{2}\right)\right] \\
& \times \exp \left\{\frac{i}{\hbar} \int_{t}^{t_{2}} \mathrm{~d} t_{3}\left[H_{22}\left(t_{3}\right)-H_{11}\left(t_{3}\right)\right]\right\} .
\end{aligned}
$$

Here, cc stands for the complex conjugate, and the matrix elements are defined as $V_{m n}(t) \equiv\langle m|V(\mathbf{r}, t)| n\rangle$ and $H_{m n}(t)$ $\equiv\langle m|H(t)| n\rangle$. By substituting Eq. (3b) into Eq. (3a) and taking the statistical average (denoted as $\langle\cdots\rangle$ ) over the plasma configurations in thermal equilibrium, we obtain a formula,

$$
\begin{aligned}
\left\langle\rho_{22}(t)\right\rangle= & -\frac{1}{\hbar^{2}} \int_{0}^{t} \mathrm{~d} t_{1} \int_{0}^{t_{1}} \mathrm{~d} t_{2}\left\langle V_{12}\left(t_{1}\right) V_{21}\left(t_{2}\right)\right. \\
& \times\left[\rho_{22}\left(t_{2}\right)-\rho_{11}\left(t_{2}\right)\right] \\
& \left.\times \exp \left\{\frac{i}{\hbar} \int_{t_{1}}^{t_{2}} \mathrm{~d} t_{3}\left[H_{22}\left(t_{3}\right)-H_{11}\left(t_{3}\right)\right]\right\}\right)+\mathrm{cc} .
\end{aligned}
$$

As long as the transition is weak, we may assume that $\rho_{22}\left(t_{2}\right) \approx 0$ and $\rho_{11}\left(t_{2}\right) \approx 1$ on the right-hand side of Eq. (4); this procedure is equivalent to the use of first-order perturbation theory. The transition rate per unit time, $w_{21}$, may then be calculated in accordance with $w_{21}$ $=\lim _{t \rightarrow \infty}(d / d t)\left\langle\rho_{22}(t)\right\rangle$. Thus,

$$
\begin{aligned}
w_{21}= & \lim _{t \rightarrow \infty} \frac{1}{\hbar^{2}} \int_{0}^{t} \mathrm{~d} t_{2}\left\langle V_{12}(t) V_{21}\left(t_{2}\right)\right. \\
& \left.\times \exp \left\{\frac{i}{\hbar} \int_{t}^{t_{2}} \mathrm{~d} t_{3}\left[H_{22}\left(t_{3}\right)-H_{11}\left(t_{3}\right)\right]\right\}\right\rangle+\mathrm{cc} .
\end{aligned}
$$

Here, the off-diagonal matrix elements, $V_{21}$ and $V_{12}$, account for the transition between the two states, while the diagonal elements, $H_{11}$ and $H_{22}$, represent the ionic energy levels for states $|1\rangle$ and $|2\rangle$, respectively. The values of $H_{11}$ and $H_{22}$ generally differ from those for an isolated ion in vacuum, because the ion is perturbed by the surrounding plasma. Such energy level shifts were ignored in our previous paper. ${ }^{1}$ To proceed further, we decompose $V(\boldsymbol{r}, t)$ into statically averaged part $\langle V(\boldsymbol{r})\rangle$ and the fluctuation $\delta V(\boldsymbol{r}, t)$ around the average, that is,

$$
V(\boldsymbol{r}, t)=\langle V(\boldsymbol{r})\rangle+\delta V(\boldsymbol{r}, t),
$$

with

$$
\begin{aligned}
& \langle V(\boldsymbol{r})\rangle=\sum_{\sigma=e, i} \frac{1}{\Omega} \sum_{\boldsymbol{k}}\left(-Z_{\sigma}\right) v(k) \exp (i \boldsymbol{k} \cdot \boldsymbol{r})\left\langle\rho_{\sigma}(\boldsymbol{k})\right\rangle \\
& \delta V(\boldsymbol{r}, t)=\sum_{\sigma=e, i} \frac{1}{\Omega} \sum_{\boldsymbol{k}}\left(-Z_{\sigma}\right) v(k) \exp (i \boldsymbol{k} \cdot \boldsymbol{r}) \delta \boldsymbol{\rho}_{\sigma}(\boldsymbol{k}, t) .
\end{aligned}
$$

Equation (7a) describes the spherically symmetric potential arising from average plasma polarization, ${ }^{5,8}$ while Eq. (7b) accounts for the potential due to time-varying plasma fluctuations and is generally non-spherical in a short time scale. Equation (5) can then be rewritten as

$$
\begin{aligned}
w_{21}= & \lim _{t \rightarrow \infty} \frac{1}{\hbar^{2}} \int_{0}^{t} \mathrm{~d} t_{2} \sum_{\sigma_{1}} \frac{1}{\sigma_{2}=e, i} \sum_{\mathbf{k}_{1} \mathbf{k}_{2}} Z_{\sigma_{1}} Z_{\sigma_{2}} v\left(k_{1}\right) v\left(k_{2}\right) \\
& \times\left\langle 1\left|\exp \left(i \mathbf{k}_{1} \cdot \mathbf{r}\right)\right| 2\right\rangle\left\langle 2\left|\exp \left(i \mathbf{k}_{2} \cdot \mathbf{r}\right)\right| 1\right\rangle \\
& \times\left\langle\delta \rho _ { \sigma _ { 1 } } ( \mathbf { k } _ { 1 } , t ) \delta \rho _ { \sigma _ { 2 } } ( \mathbf { k } _ { 2 } , t _ { 2 } ) \operatorname { e x p } \left\{\frac{i}{\hbar \Omega} \sum_{\mathbf{k}_{3}} d_{21}\left(\mathbf{k}_{3}\right)\right.\right. \\
& \left.\left.\times \sum_{\sigma_{3}=e, i}\left(-Z_{\sigma_{3}}\right) v\left(k_{3}\right) \int_{t}^{t_{2}} \mathrm{~d} t_{3} \delta \rho_{\sigma_{3}}\left(\mathbf{k}_{3}, t_{3}\right)\right\}\right\rangle \\
& \times \exp \left[i \omega_{21}\left(t_{2}-t\right)\right]+\mathrm{cc},
\end{aligned}
$$

with $d_{21}(\mathbf{k}) \equiv\langle 2|\exp (i \mathbf{k} \cdot \mathbf{r})| 2\rangle-\langle 1|\exp (i \mathbf{k} \cdot \mathbf{r})| 1\rangle$. Here, the quantity

$$
\begin{aligned}
\omega_{21}= & \frac{1}{\hbar}\left\{\left\langle 2\left|\frac{-\hbar^{2}}{2 m_{e}} \nabla^{2}-\frac{Z e^{2}}{r}+\langle V(\mathbf{r})\rangle\right| 2\right\rangle-\langle 1| \frac{-\hbar^{2}}{2 m_{e}} \nabla^{2}\right. \\
& \left.-\frac{Z e^{2}}{r}+\langle V(\mathbf{r})\rangle|1\rangle\right\},
\end{aligned}
$$


corresponds to the excitation frequency with inclusion of the energy level shift due to Eq. (7a) (plasma polarization shift).

Throughout this paper, we assume that both the electrons and ions in the plasma can be treated with the classical statistics, and that the plasma is weakly coupled in a sense that the average Coulomb interaction energy does not exceed the average thermal energy. In such a case, the random-phase approximation (RPA) is applicable to the descriptions of the Coulomb correlations. ${ }^{7}$ In RPA, one can evoke the "superposition principle," ${ }^{16}$ in which the system of interacting particles can be interpreted as a collection of noninteracting dressed particles, each wearing a screening cloud around itself. The density fluctuation operators $\delta \rho_{\sigma}(\mathbf{k}, t)$ in TCP can thus be expressed as ${ }^{7}$

$$
\begin{aligned}
\delta \rho_{e}(\mathbf{k}, t)= & \sum_{j=1}^{N_{e}} \frac{\epsilon_{i}^{(0)}\left(k, \mathbf{k} \cdot \mathbf{v}_{j}^{(e)}\right)}{\epsilon^{(0)}\left(k, \mathbf{k} \cdot \mathbf{v}_{j}^{(e)}\right)} \exp \left\{-i \mathbf{k} \cdot\left[\mathbf{r}_{j}^{(e)}(0)\right.\right. \\
& \left.\left.+\mathbf{v}_{j}^{(e)} t\right]\right\}+\sum_{j=1}^{N_{i}} Z_{\mathrm{i}} \frac{\epsilon_{e}^{(0)}\left(k, \mathbf{k} \cdot \mathbf{v}_{j}^{(i)}\right)-1}{\epsilon^{(0)}\left(k, \mathbf{k} \cdot \mathbf{v}_{j}^{(i)}\right)} \\
& \times \exp \left\{-i \mathbf{k} \cdot\left[\mathbf{r}_{j}^{(i)}(0)+\mathbf{v}_{j}^{(i)} t\right]\right\}, \\
\delta \rho_{i}(\mathbf{k}, t)= & \sum_{j=1}^{N_{i}} \frac{1}{Z_{\mathrm{i}}} \frac{\epsilon_{i}^{(0)}\left(k, \mathbf{k} \cdot \mathbf{v}_{j}^{(e)}\right)-1}{\epsilon^{(0)}\left(k, \mathbf{k} \cdot \mathbf{v}_{j}^{(e)}\right)} \exp \left\{-i \mathbf{k} \cdot\left[\mathbf{r}_{j}^{(e)}(0)\right.\right. \\
& \left.\left.+\mathbf{v}_{j}^{(e)} t\right]\right\}+\sum_{j=1}^{N_{e}} \frac{\epsilon_{e}^{(0)}\left(k, \mathbf{k} \cdot \mathbf{v}_{j}^{(i)}\right)}{\epsilon^{(0)}\left(k, \mathbf{k} \cdot \mathbf{v}_{j}^{(i)}\right)} \\
& \times \exp \left\{-i \mathbf{k} \cdot\left[\mathbf{r}_{j}^{(i)}(0)+\mathbf{v}_{j}^{(i)} t\right]\right\} .
\end{aligned}
$$

Here, $\mathbf{r}_{j}^{(\sigma)}(0)$ and $\mathbf{v}_{j}^{(\sigma)}$ refer to the initial position and velocity, respectively, of the $j$ th noninteracting particle of species $\sigma$. The function

$$
\epsilon_{\sigma}^{(0)}(k, \omega)=1+\frac{k_{\sigma}^{2}}{k^{2}} W\left(\frac{\omega / \omega_{\sigma}}{k / k_{\sigma}}\right)
$$

represents the wave number $(k)$ - and frequency $(\omega)$ dependent dielectric response function for noninteracting particles of species $\sigma, \omega_{\sigma}=\sqrt{4 \pi\left(Z_{\sigma} e\right)^{2} n_{\sigma} / m_{\sigma}}$ and $k_{\sigma}$ $=\sqrt{4 \pi\left(Z_{\sigma} e\right)^{2} n_{\sigma} / k_{\mathrm{B}} T}$ are the plasma frequency and the Debye wave number, respectively,

$$
\begin{aligned}
W(x)= & 1-x \exp \left(-\frac{x^{2}}{2}\right) \int_{0}^{x} \mathrm{~d} y \exp \left(\frac{y^{2}}{2}\right) \\
& +i \sqrt{\frac{\pi}{2}} x \exp \left(-\frac{x^{2}}{2}\right)
\end{aligned}
$$

is the $W$ function, ${ }^{7}$ and

$$
\epsilon^{(0)}(k, \omega)=1+\frac{k_{e}^{2}}{k^{2}} W\left(\frac{\omega / \omega_{e}}{k / k_{e}}\right)+\frac{k_{i}^{2}}{k^{2}} W\left(\frac{\omega / \omega_{i}}{k / k_{i}}\right)
$$

is the total dielectric function of the TCP.

The characteristic time scale of electron-density fluctuations is expected to be much smaller than that of the iondensity fluctuations, because of the large difference in the electron and ion masses. In dense TCP, however, these fluctuations cannot be separated owing to the electron-ion correlation and, as indicated in Eq. (10a), $\delta \rho_{e}(\mathbf{k}, t)$ involves the slowly fluctuating component associated with the ion motion. Accordingly, we should consider the ion-related part in both Eqs. (10a) and (10b) as the origin of the quasistatic electric microfields.

In light of the RPA expressions (10), the statistical average may be executed through integrations over the initial positions and velocities of the non-interacting particles; thus, for a general physical quantity $X$,

$$
\begin{aligned}
\langle X\rangle \equiv & \frac{1}{\Omega^{N_{e}+N_{i}}} \int d \mathbf{r}_{1}^{(e)}(0) \cdots \int d \mathbf{r}_{N_{e}}^{(e)}(0) \int d \mathbf{v}_{1}^{(e)} F_{e}\left(\mathbf{v}_{1}^{(e)}\right) \cdots \\
& \times \int d \mathbf{v}_{N_{e}}^{(e)} F_{e}\left(\mathbf{v}_{N_{e}}^{(e)}\right) \\
& \times \int d \mathbf{r}_{1}^{(i)}(0) \cdots \int d \mathbf{r}_{N_{i}}^{(i)}(0) \int d \mathbf{v}_{1}^{(i)} F_{i}\left(\mathbf{v}_{1}^{(i)}\right) \cdots \\
& \times \int d \mathbf{v}_{N_{i}}^{(i)} F_{i}\left(\mathbf{v}_{N_{i}}^{(i)}\right) X
\end{aligned}
$$

where $F_{e}(\mathbf{v})$ and $F_{i}(\mathbf{v})$ designate the Maxwell velocity distribution functions for the plasma electrons and ions, respectively: $F_{\sigma}(\mathbf{v})=\left(m_{\sigma} / 2 \pi k_{\mathrm{B}} T\right)^{3 / 2} \exp \left(-m_{\sigma} v^{2} / 2 k_{\mathrm{B}} T\right)$.

Equation (8) in conjunction with Eqs. (10) provide the general expression for the excitation rate. We remark in Eq. (8) that the term $\delta \rho_{\sigma_{1}}\left(\mathbf{k}_{1}, t\right) \delta \rho_{\sigma_{2}}\left(\mathbf{k}_{2}, t_{2}\right)$ is related to the excitation, while the following exponential factor accounts for the energy level shifts. These two factors are mutually correlated, so rigorous evaluation of the statistical average in Eq. (8) would require numerical computations involving multidimensional integrations. In this paper, we rather confine ourselves to the study of a specific case, namely, the electron-induced excitation in the presence of a quasistatic ion microfield; a simplification of Eq. (8) is possible in this case, as we shall discuss in the next subsection.

\section{B. Electron-induced excitation rate with quasistatic ion microfield}

Hereafter, we focus on the excitation induced by the electron-density fluctuations; hence, only the term with $\sigma_{1}$ $=\sigma_{2}=e$ is retained in Eq. (8).

We treat the transitions and energy level shifts independently by neglecting their mutual correlation:

$$
\begin{aligned}
& \left\langle\delta \rho _ { e } ( \mathbf { k } _ { 1 } , t ) \delta \rho _ { e } ( \mathbf { k } _ { 2 } , t _ { 2 } ) \operatorname { e x p } \left\{\frac{i}{\hbar \Omega} \sum_{\mathbf{k}_{3}} d_{21}\left(\mathbf{k}_{3}\right)\right.\right. \\
& \left.\left.\quad \times \sum_{\sigma_{3}=e, i}\left(-Z_{\sigma_{3}}\right) v\left(k_{3}\right) \int_{t}^{t_{2}} \mathrm{~d} t_{3} \delta \rho_{\sigma_{3}}\left(\mathbf{k}_{3}, t_{3}\right)\right\}\right\rangle \\
& \approx\left\langle\delta \rho_{e}\left(\mathbf{k}_{1}, t\right) \delta \rho_{e}\left(\mathbf{k}_{2}, t_{2}\right)\right\rangle\left\langle\operatorname { e x p } \left\{\frac{i}{\hbar \Omega} \sum_{\mathbf{k}_{3}} d_{21}\left(\mathbf{k}_{3}\right)\right.\right. \\
& \left.\left.\quad \times \sum_{\sigma_{3}=e, i}\left(-Z_{\sigma_{3}}\right) v\left(k_{3}\right) \int_{t}^{t_{2}} \mathrm{~d} t_{3} \delta \rho_{\sigma_{3}}\left(\mathbf{k}_{3}, t_{3}\right)\right\}\right) .
\end{aligned}
$$

The above decoupling may be valid if the factor $\delta \rho_{e}\left(\mathbf{k}_{1}, t\right) \delta \rho_{e}\left(\mathbf{k}_{2}, t_{2}\right)$ is dominated by rapid fluctuations of the electrons and the subsequent exponential factor is dominated by slow fluctuations of the ions. The merit of approxi- 
mation (15) is that it enables us to isolate the density correlation function, which can be evaluated explicitly as $\left\langle\delta \rho_{e}\left(\mathbf{k}_{1}, t\right) \delta \rho_{e}\left(\mathbf{k}_{2}, t_{2}\right)\right\rangle=\delta_{\mathbf{k}_{1}+\mathbf{k}_{2}, 0} F_{\text {ee }}\left(k_{2}, t_{2}-t\right)$, with

$$
\begin{aligned}
F_{\text {ee }}\left(k, t_{2}-t\right) \equiv & \frac{1}{\Omega}\left\langle\delta \rho_{e}(-\mathbf{k}, t) \delta \rho_{e}\left(\mathbf{k}, t_{2}\right)\right\rangle \\
= & n_{e} \int d \mathbf{v}^{(e)} F_{e}\left(\mathbf{v}^{(e)}\right) \frac{\left|\epsilon_{i}^{(0)}\left(k, \mathbf{k} \cdot \mathbf{v}^{(e)}\right)\right|^{2}}{\left|\epsilon^{(0)}\left(k, \mathbf{k} \cdot \mathbf{v}^{(e)}\right)\right|^{2}} \\
& \times \exp \left[-i \mathbf{k} \cdot \mathbf{v}^{(e)}\left(t_{2}-t\right)\right] \\
& +n_{i} \int d \mathbf{v}^{(i)} F_{i}\left(\mathbf{v}^{(i)}\right) Z_{i}^{2} \frac{\left|\epsilon_{e}^{(0)}\left(k, \mathbf{k} \cdot \mathbf{v}^{(i)}\right)-1\right|^{2}}{\left|\epsilon^{(0)}\left(k, \mathbf{k} \cdot \mathbf{v}^{(i)}\right)\right|^{2}} \\
& \times \exp \left[-i \mathbf{k} \cdot \mathbf{v}^{(i)}\left(t_{2}-t\right)\right],
\end{aligned}
$$

being the intermediate scattering function. ${ }^{17}$ Then, the transition rate can be expressed compactly as

$$
\begin{aligned}
w_{21}= & \lim _{t \rightarrow \infty} \frac{2 \omega_{e}^{2}}{\pi Z} \int_{0}^{t} \mathrm{~d} t_{2} D_{21}\left(t_{2}-t\right) \\
& \times\left\langle\operatorname { e x p } \left\{\frac{i}{\hbar \Omega} \sum_{\mathbf{k}_{3}} d_{21}\left(\mathbf{k}_{3}\right) \sum_{\sigma_{3}=e, i} \sum_{j_{3}=1}^{N_{\sigma_{3}}} \frac{-Z_{\sigma_{3}} v\left(k_{3}\right)}{\epsilon^{(0)}\left(k_{3}, \mathbf{k}_{3} \cdot \mathbf{v}_{j_{3}}^{\left(\sigma_{3}\right)}\right)}\right.\right. \\
& \left.\left.\times \int_{t}^{t_{2}} \mathrm{~d} t_{3} \exp \left[-i \mathbf{k}_{3} \cdot\left(\mathbf{r}_{j_{3}}^{\left(\sigma_{3}\right)}(0)+\mathbf{v}_{j_{3}}^{\left(\sigma_{3}\right)} t_{3}\right)\right]\right\}\right) \\
& \times \exp \left[i \omega_{21}\left(t_{2}-t\right)\right]+\mathrm{cc} .
\end{aligned}
$$

Here the dimensionless function

$$
\begin{aligned}
D_{21}\left(t_{2}-t\right) \equiv & \int_{0}^{\infty} d\left(k a_{0}\right) \frac{\overline{|\langle 2|\exp (i \mathbf{k} \cdot \mathbf{r})| 1\rangle|^{2}}}{\left(k a_{0}\right)^{2}} \\
& \times \frac{1}{n_{e}} F_{\text {ee }}\left(k, t_{2}-t\right)
\end{aligned}
$$

represents the density correlation function integrated over $k$ with the weight of the angular-averaged generalized oscillator strength,

$$
\begin{aligned}
& \frac{\overline{|\langle 2|\exp (i \mathbf{k} \cdot \mathbf{r})| 1\rangle|^{2}}}{\left(k a_{0}\right)^{2}} \\
& \equiv \frac{1}{4 \pi} \int_{-1}^{1} d(\cos \theta) \int_{0}^{2 \pi} d \varphi \frac{|\langle 2|\exp (i \mathbf{k} \cdot \mathbf{r})| 1\rangle|^{2}}{\left(k a_{0}\right)^{2}},
\end{aligned}
$$

where the angles $\theta$ and $\varphi$ specify the direction of $\mathbf{k}$ in the spherical coordinates; $a_{0} \equiv a_{\mathrm{B}} / Z$ with $a_{\mathrm{B}}$ being the Bohr radius.

We note that the function $D_{21}\left(t_{2}-t\right)$ generally decays to zero for sufficiently large value of $\left|t_{2}-t\right|$; let $t_{\max }$ be the corresponding decay time. If an equality $\left|\mathbf{k}_{3} \cdot \mathbf{v}_{j_{3}}^{(i)} t_{\max }\right|<1$ $<\left|\mathbf{k}_{3} \cdot \mathbf{v}_{j_{3}}^{(e)} t_{\max }\right|$ holds for the $t_{3}$-integration on the right-hand side of Eq. (17), the following simplified treatments may be applicable, as a first approximation, to the electron- and ioninduced energy level shifts:

$$
\int_{t}^{t_{2}} \mathrm{~d} t_{3} \exp \left(-i \mathbf{k}_{3} \cdot \mathbf{v}_{j_{3}}^{(e)} t_{3}\right) \approx 0
$$

$$
\int_{t}^{t_{2}} \mathrm{~d} t_{3} \exp \left(-i \mathbf{k}_{3} \cdot \mathbf{v}_{j_{3}}^{(i)} t_{3}\right) \approx t_{2}-t
$$

Physically, Eq. (20a) is equivalent to neglecting the electronimpact broadening of the bound state levels, ${ }^{10,18}$ while Eq. (20b) corresponds to the quasistatic approximation for the ion microfield.

To check the validity of Eqs. (20), let us estimate the approximate value of $t_{\max }$ through formula (18). First, we notice that for typical allowed transitions, the generalized oscillator strength is almost constant for $k a_{0}<1$ and vanishes rapidly for $k a_{0}>1 .^{6}$ Second, it has been known ${ }^{17}$ that $\left.F_{\text {ee }}\left(k, t_{2}-t\right)=n_{e} \exp \left\{-\left[\left(t_{2}-t\right) v_{\text {th }}^{(e)} / \sqrt{6}\right]^{2} k^{2}\right]\right\} \quad$ for a onecomponent system of ideal-gas electrons. ${ }^{17}$ Here, $v_{\text {th }}^{(\sigma)}$ $\equiv \sqrt{3 k_{\mathrm{B}} T / m_{\sigma}}$ refers to the thermal velocity of the electrons $(\sigma=e)$ and ions $(\sigma=i)$, respectively. These observations lead us to an approximate expression, $D_{21}\left(t_{2}-t\right)$ $\approx \int_{0}^{1} d\left(k a_{0}\right) \exp \left[-\alpha^{2}\left(k a_{0}\right)^{2}\right]$, with $\alpha \equiv\left|t_{2}-t\right| v_{\text {th }}^{(e)} / \sqrt{6} a_{0}$. This integral is equal to unity for $\alpha=0$ and diminishes as $\sqrt{\pi} / 2 \alpha$ for $\alpha \gg 1$. We thus find that the value of $D_{21}\left(t_{2}-t\right)$ reaches $\frac{1}{10}$ of the initial value when $\alpha \approx 8$, which corresponds to $\mid t_{2}$ $-t \mid \approx 20 a_{0} / v_{\mathrm{th}}^{(e)}$; when the latter value is adopted for $t_{\max }$, we estimate that $\left|\mathbf{k}_{3} \cdot \mathbf{v}_{j_{3}}^{(i)} t_{\max }\right| \approx 20 v_{\text {th }}^{(i)} / v_{\text {th }}^{(e)} \approx 0.5<1$ and $\mid \mathbf{k}_{3}$ $\cdot \mathbf{v}_{j_{3}}^{(e)} t_{\max } \mid \approx 20>1$ for hydrogen plasmas. Here, we have adopted $k_{3} \approx 1 / a_{0}$, for which the function $d_{21}\left(\mathbf{k}_{3}\right)$ takes on the largest value. Validity of Eqs. (20) has thereby been indicated.

We remark, however, that the function $D_{21}\left(t_{2}-t\right)$ consists of both electron and ion terms, as indicated by Eq. (16). The above argument may be valid as long as the electronrelated term makes a dominant contribution to the excitation rate. The ion-related term in Eq. (16) plays a role for transitions between closely spaced levels at high plasma densities, as we shall see later in Sec. IIIC and also shown in the previous papers ${ }^{1,3}$. For this ion-related contribution, dynamical effects beyond Eq. (20b) would come into play in the level shift; moreover, the corresponding transitions and level shifts cannot be treated independently, because Eq. (15) may also break down. These are the problems beyond the scope of the present work.

In this way, we arrive at a formula of electron-induced excitation rate,

$$
\begin{aligned}
w_{21}= & \lim _{t \rightarrow \infty} \frac{1}{\hbar^{2}} \frac{1}{\Omega} \sum_{\mathbf{k}}|v(k)|^{2}|\langle 2|\exp (i \mathbf{k} \cdot \mathbf{r})| 1\rangle|^{2} \\
& \times \int_{0}^{t} \mathrm{~d} t_{2} F_{\mathrm{ee}}\left(k, t_{2}-t\right) \\
& \times\left\langle\exp \left[i\left(\omega_{21}+\Delta \omega_{21}\right)\left(t_{2}-t\right)\right]\right\rangle+\mathrm{cc} \\
= & \frac{2 \pi}{\hbar^{2} \Omega} \sum_{\mathbf{k}}|v(k)|^{2}|\langle 2|\exp (i \mathbf{k} \cdot \mathbf{r})| 1\rangle|^{2} \\
& \times\left\langle S_{\mathrm{ee}}\left(k, \omega_{21}+\Delta \omega_{21}\right)\right\rangle,
\end{aligned}
$$

with the energy level shift due to the ion microfield being taken into account through 


$$
\begin{aligned}
& \Delta \omega_{21}=\frac{1}{\hbar}[\langle 2|\Phi(\mathbf{r})| 2\rangle-\langle 1|\Phi(\mathbf{r})| 1\rangle] \\
& \Phi(\mathbf{r})=\sum_{j=1}^{N_{i}} \frac{1}{\Omega} \sum_{\mathbf{k}} \frac{-Z_{\mathrm{i}} v(k)}{\epsilon^{(0)}\left(k, \mathbf{k} \cdot \mathbf{v}_{j}^{(i)}\right)} \exp \left[i \mathbf{k} \cdot\left(\mathbf{r}-\mathbf{r}_{j}^{(i)}(0)\right)\right] .
\end{aligned}
$$

The quantity $S_{\text {ee }}(k, \omega)$ is the electron-electron dynamical structure factor in the RPA, 1,7

$$
\begin{aligned}
S_{\mathrm{ee}}(k, \omega) \equiv & \frac{1}{2 \pi} \int_{-\infty}^{\infty} d t F_{\mathrm{ee}}(k, t) \exp (i \omega t) \\
= & \frac{\left|\epsilon_{i}^{(0)}(k, \omega)\right|^{2}}{\left|\epsilon^{(0)}(k, \omega)\right|^{2}} S_{\mathrm{ee}}^{(0)}(k, \omega) \\
& +Z_{i}^{2} \frac{\left|\epsilon_{e}^{(0)}(k, \omega)-1\right|^{2}}{\left|\epsilon^{(0)}(k, \omega)\right|^{2}} S_{\mathrm{ii}}^{(0)}(k, \omega),
\end{aligned}
$$

with

$$
S_{\sigma \sigma}^{(0)}(k, \omega)=\sqrt{\frac{m_{\sigma}}{2 \pi k_{\mathrm{B}} T}} \frac{n_{\sigma}}{k} \exp \left(-\frac{m_{\sigma} \omega^{2}}{2 k_{\mathrm{B}} T k^{2}}\right) .
$$

It is clear that $\Phi(\mathbf{r})$ describes the interaction of the bound electron with the quasistatic electric microfield produced by $N_{i}$ ions located at $\mathbf{r}_{j}^{(i)}(0)\left(j=1, \cdots, N_{i}\right)$, and $\Delta \omega_{21}$ represents the resultant shift of the excitation frequency. It is notable that $\Phi(\mathbf{r})$ involves the total dielectric function $\boldsymbol{\epsilon}^{(0)}(k, \mathbf{k}$ $\left.\cdot \mathbf{v}_{j}^{(i)}\right)$, which accounts for the screening of the ion microfield. This does not necessarily mean that both electrons and ions actually participate in the screening, because the efficiency of screening depends on the magnitude of $\mathbf{k}$ $\cdot \mathbf{v}_{j}^{(i)}$. It follows from Eq. (11) that ${ }^{3,5}$

$$
\begin{aligned}
& \boldsymbol{\epsilon}^{(0)}\left(k, \mathbf{k} \cdot \mathbf{v}_{j}^{(i)}\right) \\
& \approx\left\{\begin{array}{lll}
1, & \text { for } & \mathbf{k} \cdot \mathbf{v}_{j}^{(i)} \gg \omega_{e} \gg \omega_{i}, \\
\epsilon_{e}^{(0)}(k, 0), & \text { for } & \omega_{e} \gg \mathbf{k} \cdot \mathbf{v}_{j}^{(i)} \gg \omega_{i}, \\
\epsilon^{(0)}(k, 0), & \text { for } & \omega_{e} \gg \omega_{i} \gg \mathbf{k} \cdot \mathbf{v}_{j}^{(i)} .
\end{array}\right.
\end{aligned}
$$

Owing to the factor $d_{21}(\mathbf{k})$ in Eq. (17), the $k$ value close to $a_{0}^{-1}$ gives the dominant contribution to the energy level shift; it may also be reasonable to assume that $v_{j}^{(i)} \approx v_{\text {th }}^{(i)}$. Then, it turns out that $\mathbf{k} \cdot \mathbf{v}_{j}^{(i)}$ is virtually independent of the plasma densities. On the other hand, $\omega_{e}$ and $\omega_{i}$ are increasing functions of the plasma densities. Consequently, at fixed temperature, Eq. (26a) tends to be satisfied at relatively low densities while Eq. (26c) may be realized at fairly high densities.

For tightly bound states, the electric microfield varies only slightly within the spatial dimension $\left(\approx a_{0}\right)$ of the electron orbitals in the target ion. Therefore, it is reasonable ${ }^{13}$ to expand $\Phi(\mathbf{r})$ as $\Phi(\mathbf{r}) \approx \Phi(0)+\mathbf{r} \cdot \nabla \Phi(0)$ in Eq. (22); the leading monopole term does not contribute to $\Delta \omega_{21}$, and we have

$$
\Delta \omega_{21}(\mathcal{E})=\frac{1}{\hbar}[\langle 2|e \mathcal{E} \cdot \mathbf{r}| 2\rangle-\langle 1|e \mathcal{E} \cdot \mathbf{r}| 1\rangle],
$$

with $\mathcal{E}=\boldsymbol{\nabla} \Phi(0) / e$ describing the local electric field at the position of the target ion. Equation (27) thus gives the energy level shift due to the linear Stark effect. ${ }^{15,19,20}$
Once the dependence of $\Phi(\mathbf{r})$ on the ion velocity $\mathbf{v}_{j}^{(i)}$ is eliminated by replacing $\epsilon^{(0)}\left(k, \mathbf{k} \cdot \mathbf{v}_{j}^{(i)}\right)$ by its suitable static counterpart as shown in Eqs. (26), we can introduce the distribution function $P(\mathcal{E})$ of field strengths ${ }^{14,18}$ according to

$$
\begin{aligned}
P(\mathcal{E})= & 4 \pi \mathcal{E}^{2} \frac{1}{\Omega^{N_{i}}} \int d \mathbf{r}_{1}^{(i)}(0) \cdots \int d \mathbf{r}_{N_{i}}^{(i)}(0) \\
& \times \delta[\mathcal{E}-\nabla \Phi(0) / e],
\end{aligned}
$$

which satisfies $\int_{0}^{\infty} d \mathcal{E} P(\mathcal{E})=1$. This function plays a central role in line-broadening theories of plasmas. ${ }^{14,18}$ The field strength in a plasma is measured by the Holtsmark's characteristic field strength ${ }^{18}$ defined through

$$
\mathcal{E}_{0} \equiv\left(\frac{8 \pi}{25}\right)^{1 / 3} \frac{Z_{\mathrm{i}} e}{a^{2}}
$$

with $a \equiv\left(3 Z_{\mathrm{i}} / 4 \pi n_{e}\right)^{1 / 3}$ being the ion-sphere radius. It has been known ${ }^{14,18}$ that $P(\mathcal{E})$ exhibits a single broad peak at $\mathcal{E}$ $\simeq 1.6 \mathcal{E}_{0}$ in the weak-coupling limit $\Gamma \equiv\left(Z_{i} e\right)^{2} / a k_{\mathrm{B}} T \rightarrow 0$; the peak becomes sharper as $\Gamma$ increases, and the peak position is located at $\mathcal{E} \simeq \mathcal{E}_{0}$ for $\Gamma=0.5$. ${ }^{14}$ In terms of $P(\mathcal{E})$, we obtain the final expression for the excitation rate,

$$
\begin{aligned}
w_{21}= & \int_{0}^{\infty} d \mathcal{E} P(\mathcal{E}) \frac{2 \pi}{\hbar^{2} \Omega} \sum_{\mathbf{k}}|v(k)|^{2}|\langle 2|\exp (i \mathbf{k} \cdot \mathbf{r})| 1\rangle|^{2} \\
& \times S_{\mathrm{ee}}\left[k, \omega_{21}+\Delta \omega_{21}(\mathcal{E})\right] \\
= & \int_{0}^{\infty} d \mathcal{E} P(\mathcal{E}) \frac{4 \omega_{e}}{Z} \int_{0}^{\infty} d\left(k a_{0}\right) \frac{\overline{|\langle 2|\exp (i \mathbf{k} \cdot \mathbf{r})| 1\rangle|^{2}}}{\left(k a_{0}\right)^{2}} \\
& \times \frac{\omega_{e}}{n_{e}} S_{\mathrm{ee}}\left[k, \omega_{21}+\Delta \omega_{21}(\mathcal{E})\right] .
\end{aligned}
$$

We summarize the salient feature in Eq. (30) below. The electron-induced transition rate is governed by the electronelectron dynamical structure factor of the plasma evaluated at the frequency equal to the transition frequency between the initial and final states. This transition frequency involves the level shift due to average plasma polarization and that due to quasistatic electric microfield of the ions; the latter contribution, $\Delta \omega_{21}$, depends on the magnitude $\mathcal{E}$ of instantaneous electric field at the position of the target ion during the transition. Average over $\mathcal{E}$ is taken at the final stage of the calculation with the aid of the distribution function $P(\mathcal{E})$. Formula (30) embodies the concept of the microfield stochastic model proposed by Murillo. ${ }^{13}$

\section{NUMERICAL EXAMPLES}

In this section, the effect of energy levels shifts on the electron-induced $2 s_{1 / 2} \rightarrow 2 p_{3 / 2}$ and $2 p_{1 / 2} \rightarrow 2 p_{3 / 2}$ finestructure transitions of a $\mathrm{Ne}^{9+}$ ion $(Z=10)$ in hydrogen plasmas $\left(Z_{\mathrm{i}}=1\right)$ is investigated as a concrete example. The plasma temperature is fixed at $k_{\mathrm{B}} T=340 \mathrm{eV}$; the plasma density $n_{e}$ is varied from $10^{20}$ to $10^{23} \mathrm{~cm}^{-3}$. Even at the highest density, the Coulomb coupling parameter $\Gamma$ of the plasma $^{7}$ remains smaller than unity $(\Gamma=0.03)$ so that the use of RPA is valid. 


\section{A. Stark effect}

To obtain the energy eigenvalues and eigenfunctions for the fine-structure levels of a hydrogen-like ion under a constant electric field $\mathcal{E}$, we follow the earlier calculation by Lüders ${ }^{19}$ based on the degenerate state perturbation theory. When $\mathcal{E}=0$, we have four relevant states, each of which is doubly degenerate:

$$
\begin{aligned}
& \left|2 p_{3 / 2}, M= \pm 3 / 2\right\rangle=|21 \pm 1\rangle| \pm 1 / 2\rangle, \\
& \left|2 p_{3 / 2}, M= \pm 1 / 2\right\rangle=\sqrt{\frac{1}{3}}|21 \pm 1\rangle|\mp 1 / 2\rangle \\
& +\sqrt{\frac{2}{3}}|2 \quad 10\rangle| \pm 1 / 2\rangle, \\
& \left|2 p_{1 / 2}, M= \pm 1 / 2\right\rangle=\sqrt{\frac{2}{3}}|21 \pm 1\rangle|\mp 1 / 2\rangle \\
& -\sqrt{\frac{1}{3}}|2 \quad 10\rangle| \pm 1 / 2\rangle, \\
& \left|2 s_{1 / 2}, M= \pm 1 / 2\right\rangle=|200\rangle| \pm 1 / 2\rangle .
\end{aligned}
$$

Here, $M$ designates the total magnetic quantum number; the eigenkets on the right-hand side should be interpreted as $|n l m\rangle\left|m_{s}\right\rangle$, where $n, l, m$ are the principal, azimuthal, and magnetic quantum numbers, respectively; $m_{s}$ specifies the $z$ component of the electron spin. The energy of states (31a) and (31b) lies above that of states (31c) and (31d) by an amount, (1/16) $\alpha^{2} Z^{4}$ Ry ( $\left.=0.45 \mathrm{eV}\right)$, with $\mathrm{Ry} \equiv e^{2} / 2 a_{\mathrm{B}}$ and $\alpha$ being the fine-structure constant; this corresponds to the field-free fine-structure splitting. ${ }^{15}$ The tiny splitting between $2 s_{1 / 2}$ and $2 p_{1 / 2}$ states due to Lamb shift ${ }^{15}$ is neglected here.

When the electric field is applied, the energy of state (31a) remains unchanged; let us denote this unshifted state simply as $|0\rangle$ and fix its energy at zero. The remaining three states (31b)-(31d) are mixed together to form three Stark components, which will be denoted as $|U\rangle$ (upper state), $|M\rangle$ (middle state), and $|L\rangle$ (lower state): The corresponding eigenenergies $\xi_{U}, \xi_{M}$, and $\xi_{L}$, measured in units of $\alpha^{2} Z^{4} \mathrm{Ry}$, are obtained ${ }^{19}$ through solutions to the secular equation: ${ }^{20}$

$$
\xi_{i}^{3}+\frac{1}{8} \xi_{i}^{2}+\left(\frac{1}{256}-9 A^{2}\right) \xi_{i}-\frac{3}{8} A^{2}=0 \quad(i=U, M, L),
$$

where

$$
A \equiv \frac{e \mathcal{E} a_{0}}{\alpha^{2} Z^{4} \mathrm{Ry}}=\left(\frac{8 \pi}{25}\right)^{1 / 3} \frac{2 Z_{\mathrm{i}}}{\alpha^{2} Z^{5}}\left(\frac{a_{\mathrm{B}}}{a}\right)^{2} \frac{\mathcal{E}}{\mathcal{E}_{0}},
$$

is the dimensionless electric field strength. The value of $A$ increases as the plasma density increases. It follows from the third term on the left-hand side of Eq. (32) that the condition $A<1 / 48$ provides the criterion of the weak-field regime where the magnitude of Stark splitting is small compared with the field-free fine-structure splitting; the strong-field regime corresponds to the case with $A>1 / 48$.

The energy eigenvalues of the four sublevels calculated for $\mathcal{E}=\mathcal{E}_{0}$ [Eq. (28)] are shown by the dotted curves in Fig. 1. The crossover from weak- to strong-field regime occurs at $n_{e}=2.1 \times 10^{22} \mathrm{~cm}^{-3}$.

Because of the Stark mixing, the corresponding eigenfunctions are expressed as linear combinations of the unperturbed eigenfunctions (31b)-(31d) as

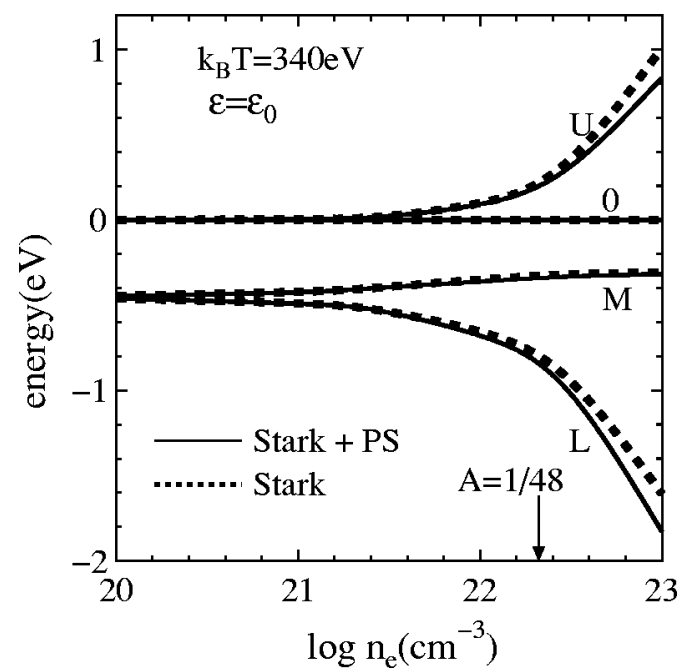

FIG. 1. Energy eigenvalues for the $n=2$ Stark components of a $\mathrm{Ne}^{9+}$ ion in a hydrogen TCP of $k_{\mathrm{B}} T=340 \mathrm{eV}$, plotted against plasma density; $\mathcal{E}=\mathcal{E}_{0}$ is assumed. The solid and dotted curves represent the results with and without the plasma polarization shifts (39), respectively. The energy of state $|0\rangle$ is fixed at zero.

$$
\begin{aligned}
|i\rangle= & c_{2 i}\left|2 p_{3 / 2}, M= \pm 1 / 2\right\rangle+c_{3 i}\left|2 p_{1 / 2}, M= \pm 1 / 2\right\rangle \\
& +c_{4 i}\left|2 s_{1 / 2}, M= \pm 1 / 2\right\rangle \quad(i=U, M, L), \\
|0\rangle= & \left|2 p_{3 / 2}, M= \pm 3 / 2\right\rangle,
\end{aligned}
$$

where the coefficients are given by

$$
\begin{aligned}
c_{2 i} & =-\frac{\sqrt{6} A}{\xi_{i}} c_{4 i}, \quad c_{3 i}=\frac{\sqrt{3} A}{\frac{1}{16}+\xi_{i}} c_{4 i}, \\
c_{4 i} & =\left[\frac{6 A^{2}}{\xi_{i}^{2}}+\frac{3 A^{2}}{\left(\frac{1}{16}+\xi_{i}\right)^{2}}+1\right]^{-1 / 2} \quad(i=U, M, L) .
\end{aligned}
$$

In the limit of $A \rightarrow 0$, where $\xi_{U}=\xi_{0}=0$ and $\xi_{M}$ $=\xi_{L}=-1 / 16$, the values of the mixing coefficients are $\left(c_{2 U}, c_{3 U}, c_{4 U}\right)=(-1,0,0), \quad\left(c_{2 M}, c_{3 M}, c_{4 M}\right)$ $=(0,1 / \sqrt{2}, 1 / \sqrt{2}), \quad\left(c_{2 L}, c_{3 L}, c_{4 L}\right)=(0,-1 / \sqrt{2}, 1 / \sqrt{2})$. In the limit of strong field, $A \rightarrow \infty$, the corresponding values are $\left(c_{2 U}, c_{3 U}, c_{4 U}\right)=(-1 / \sqrt{3}, 1 / \sqrt{6}, 1 / \sqrt{2}), \quad\left(c_{2 M}, c_{3 M}, c_{4 M}\right)$ $=(1 / \sqrt{3}, \sqrt{2 / 3}, 0),\left(c_{2 L}, c_{3 L}, c_{4 L}\right)=(1 / \sqrt{3},-1 / \sqrt{6}, 1 / \sqrt{2})$.

The generalized oscillator strengths which enter Eq. (30) can be explicitly calculated by using the hydrogenic wave functions, ${ }^{21}$ as explained briefly in the Appendix. They are expressed in terms of the mixing coefficients (35) as

$$
\begin{aligned}
\frac{\overline{|\langle i|\exp (i \mathbf{k} \cdot \mathbf{r})| j\rangle|^{2}}}{K^{2}}= & \alpha_{i j} \frac{K^{2}}{\left(K^{2}+1\right)^{8}}+\beta_{i j} \frac{\left(K^{2}-1\right)^{2}}{\left(K^{2}+1\right)^{8}} \\
& (i, j=U, M, L), \\
\frac{|\langle 0|\exp (i \mathbf{k} \cdot \mathbf{r})| j\rangle|^{2}}{K^{2}}= & \alpha_{0 j} \frac{K^{2}}{\left(K^{2}+1\right)^{8}}+\beta_{0 j} \frac{\left(K^{2}-1\right)^{2}}{\left(K^{2}+1\right)^{8}} \\
& (j=U, M, L),
\end{aligned}
$$

with $K \equiv k a_{0}$, and 


$$
\begin{aligned}
\alpha_{i j}= & \frac{4}{5}\left[c_{2 i} c_{2 j}-\sqrt{2}\left(c_{2 i} c_{3 j}+c_{3 i} c_{2 j}\right)\right]^{2}+4 c_{4 i}^{2} c_{4 j}^{2} \\
& +\frac{4}{15}\left[2 \sqrt{2}\left(c_{2 i} c_{2 j}-c_{3 i} c_{3 j}\right)+c_{2 i} c_{3 j}+c_{3 i} c_{2 j}\right]^{2} \\
\alpha_{0 j}= & \frac{4}{5}\left(\sqrt{2} c_{2 j}-c_{3 j}\right)^{2}+\frac{8}{5}\left(c_{2 j}+\sqrt{2} c_{3 j}\right)^{2}, \\
\beta_{i j}= & \frac{1}{3}\left[-\sqrt{6}\left(c_{2 i} c_{4 j}+c_{4 i} c_{2 j}\right)+\sqrt{3}\left(c_{3 i} c_{4 j}+c_{4 i} c_{3 j}\right)\right]^{2} \\
& +2\left[\frac{1}{\sqrt{2}}\left(c_{4 i} c_{2 j}-c_{2 i} c_{4 j}\right)+c_{4 i} c_{3 j}-c_{3 i} c_{4 j}\right]^{2} \\
\beta_{0 j}= & 3 c_{4 j}^{2} .
\end{aligned}
$$

In Eqs. (36), the first term on the right-hand side involving $\alpha_{i j}$ or $\alpha_{0 j}$ vanishes in the limit of $K \rightarrow 0$ and corresponds to the quadrupole transition, while the second term involving $\beta_{i j}$ or $\beta_{0 j}$ is finite at $K=0$ and describes the dipole-allowed transition; it turns out that the latter contribution to the excitation rate is larger than the former by two orders of magnitude.

\section{B. Polarization shift}

In addition to the Stark splitting treated in the previous subsection, the energy levels are further modified through the plasma polarization shift arising from Eq. (7a). Here, the induced plasma density $\left\langle\rho_{\sigma}(\mathbf{k})\right\rangle$ on the right-hand side of Eq. (7a) is evaluated simply through the linear response of plasma electrons and ions against the Coulomb field of the target ion. ${ }^{7}$ Thus, $\langle V(\mathbf{r})\rangle=-\left(Z e^{2} / r\right)[\exp (-r / D)-1]$, with $D=\left[4 \pi e^{2} n_{e} / k_{\mathrm{B}} T+4 \pi\left(Z_{\mathrm{i}} e\right)^{2} n_{i} / k_{\mathrm{B}} T\right]^{-1 / 2}$, representing the Debye screening length. ${ }^{7}$ As long as $D \gg a_{0},\langle V(\mathbf{r})\rangle$ can be regarded as a small perturbation to the bound-state electron, and it may also be expanded as $\langle V(\mathbf{r})\rangle \approx Z e^{2} / D$ $-Z e^{2} r / 2 D^{2}$ inside the target ion. Thus, the level shift for state $|i\rangle(i=U, 0, M, L)$ may be evaluated as

$$
\begin{gathered}
\Delta E_{i}^{\mathrm{pol}}=\langle i|\langle V(\mathbf{r})\rangle| i\rangle=\frac{Z e^{2}}{D}-\frac{Z e^{2}}{2 D^{2}}\langle i|r| i\rangle \\
(i=U, 0, M, L) .
\end{gathered}
$$

The term $Z e^{2} / D$ on the right-hand side produces equal level shifts for all the bound states; it does not affect the excitation energies of bound-bound transitions, and hence, it can be neglected in this study. With the aid of Eqs. (34), we find that the frequency of the $|j\rangle \rightarrow|i\rangle$ transition is modified by an amount

$$
\begin{aligned}
\Delta \omega_{i j}^{\mathrm{pol} \equiv} \equiv & \frac{1}{\hbar}\left(\Delta E_{i}^{\mathrm{pol}}-\Delta E_{j}^{\mathrm{pol}}\right)=-\frac{1}{\hbar}\left(c_{4 i}^{2}-c_{4 j}^{2}\right)\left(\frac{a_{0}}{D}\right)^{2} Z^{2} \mathrm{Ry} \\
& (i, j=U, M, L), \\
\Delta \omega_{0 j}^{\mathrm{pol}} \equiv & \frac{1}{\hbar}\left(\Delta E_{0}^{\mathrm{pol}}-\Delta E_{j}^{\mathrm{pol}}\right)=\frac{1}{\hbar} c_{4 j}^{2}\left(\frac{a_{0}}{D}\right)^{2} Z^{2} \mathrm{Ry} \\
& (j=U, M, L) .
\end{aligned}
$$

The Stark components modified by those plasma polarization shifts are indicated by the solid curves in Fig. 1. We find that the effect of the polarization shifts is insignificant.

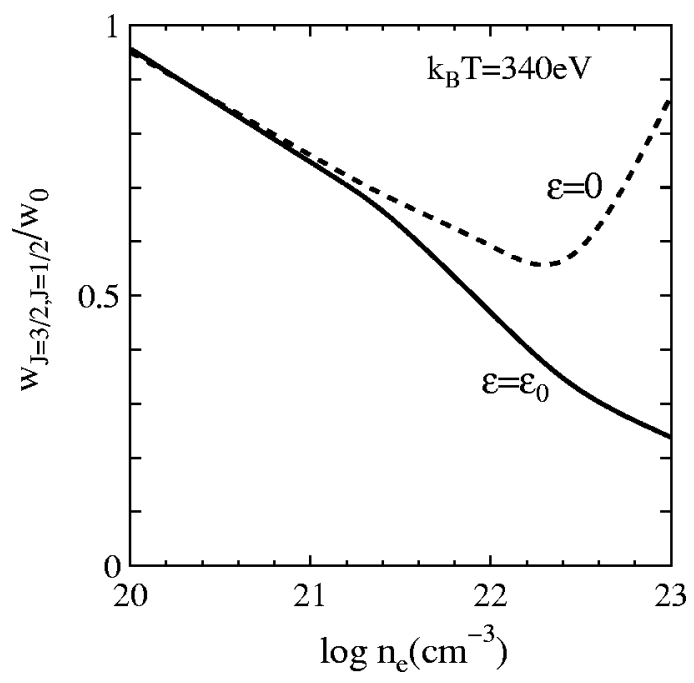

FIG. 2. Electron-induced excitation rate $w_{J=3 / 2, J=1 / 2}$ for a $\mathrm{Ne}^{9+}$ ion in a hydrogen TCP of $k_{\mathrm{B}} T=340 \mathrm{eV}$. Solid and dotted curves correspond to the cases with $\mathcal{E}=\mathcal{E}_{0}$ and $\mathcal{E}=0$, respectively. The excitation rates are normalized by the low-density value, $w_{0} \equiv 1.33 \times 10^{-8}\left[n_{e}\left(\mathrm{~cm}^{-3}\right)\right] \mathrm{s}^{-1}$.

Even at the highest plasma density, $n_{e}=10^{23} \mathrm{~cm}^{-3}$, the Debye length is fairly large $\left(D / a_{0}=58\right)$ so that the $n=2$ sublevels are virtually unaffected. ${ }^{8}$ It may not be necessary to consider the corresponding modification to the wave functions for such a weak perturbation. More advanced treatments of the polarization shifts would be required for shallow bound states and/or strongly coupled plasmas with $\Gamma>1.4,5,10$

\section{Numerical results}

By using Eq. (30), we have computed the electroninduced excitation rates $w_{i j}$ for the following transitions: $|M\rangle \rightarrow|U\rangle, \quad|L\rangle \rightarrow|U\rangle|M\rangle \rightarrow|0\rangle$, and $|L\rangle \rightarrow|0\rangle$. As explained in the previous subsections, the excitation frequencies which enter Eq. (30) are evaluated as

$$
\begin{aligned}
\omega_{i j}+\Delta \omega_{i j}(\mathcal{E})= & \frac{\alpha^{2} Z^{4}}{\hbar} \frac{e^{2}}{2 a_{\mathrm{B}}}\left(\xi_{i}-\xi_{j}\right)+\Delta \omega_{i j}^{\mathrm{pol}} \\
& (i, j=U, 0, M, L) .
\end{aligned}
$$

In Fig. 2, we plot the total excitation rate of the above four transitions, $w_{J=3 / 2, J=1 / 2} \equiv w_{U M}+w_{0 M}+w_{U L}+w_{0 L}$. In the low-density limit, where the electric microfield is negligible $(A \rightarrow 0)$, the coefficients (37) become $\alpha_{U M}=\alpha_{U L}=14 / 15$, $\alpha_{0 M}=\alpha_{0 L}=2, \quad \beta_{U M}=\beta_{U L}=\beta_{0 M}=\beta_{0 L}=3 / 2 ; \quad$ hence, $w_{J=3 / 2, J=1 / 2}$ approaches the limiting value, $w_{0} \equiv 1.33$ $\times 10^{-8}\left[n_{e}\left(\mathrm{~cm}^{-3}\right)\right] \mathrm{s}^{-1}$, which corresponds to the sum of $2 s_{1 / 2} \rightarrow 2 p_{3 / 2}$ and $2 p_{1 / 2} \rightarrow 2 p_{3 / 2}$ transition rates. This expression for $w_{0}$ can be obtained directly from Eq. (30) by neglecting the level shifts altogether and setting $\epsilon_{e}^{(0)}(k, \omega)$ $=\epsilon_{i}^{(0)}(k, \omega)=\epsilon^{(0)}(k, \omega) \equiv 1$. We remark that the $2 s_{1 / 2}$ $\rightarrow 2 p_{3 / 2}$ transition is dipole allowed, so that the transition rate, $1.31 \times 10^{-8}\left[n_{e}\left(\mathrm{~cm}^{-3}\right)\right] \mathrm{s}^{-1}$, dominates bulk of $w_{0}$; the contribution of the quadrupole $2 p_{1 / 2} \rightarrow 2 p_{3 / 2}$ transition is smaller by two orders of magnitude.

In the density range $10^{20}<n_{e}\left(\mathrm{~cm}^{-3}\right)<10^{21}$, we observe that the transition rate decreases compared to $w_{0}$ as the 


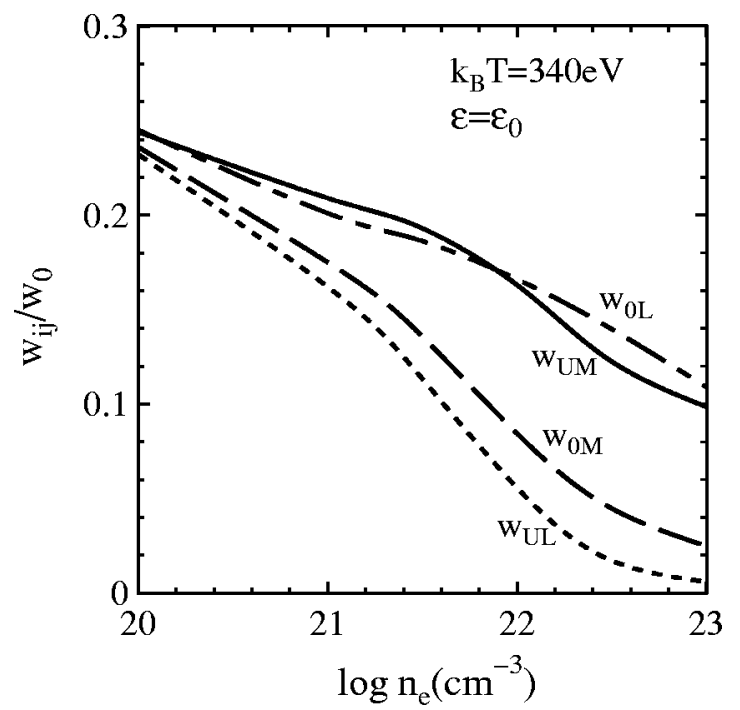

FIG. 3. Individual contributions to $w_{J=3 / 2, J=1 / 2}$ shown in Fig. 2 for $\mathcal{E}=\mathcal{E}_{0}$.

plasma density increases. This manifests the effect of dynamical screening described by the dielectric functions in Eq. (24).

The effect of energy level shifts on the excitation rate emerges at higher densities, $n_{e}>10^{21} \mathrm{~cm}^{-3}$. When the energy level shifts are neglected, the excitation rate starts to increase divergently as $n_{e}$ exceeds about $10^{22} \mathrm{~cm}^{-3}$. As reported previously, ${ }^{1,3}$ the origin of this divergence can be attributed to an increasing contribution from the the $S_{\mathrm{ii}}^{(0)}$-related term on the right-hand side of Eq. (24); this ionrelated term physically represents the low-frequency density fluctuation produced by the electrons comoving with the ions. It was mentioned in Ref. 1 that first-order perturbation theory is no longer valid in this regime since the excitation rate is too large. When the level shifts due to the quasistatic electric microfield are included, however, the excitation rate is significantly suppressed and the divergent increase no longer appears, as illustrated by the solid curve in Fig. 2.

To elucidate the reason for the reduction of the excitation rates at high densities, we plot in Fig. 3 the individual contributions $w_{U M}, w_{0 M}, w_{U L}$, and $w_{0 L}$ separately. We observe that the decrease of $w_{0 M}$ and $w_{U L}$ are particularly notable. For these transitions, the contributions of the dipole-allowed transition vanish $\left(\beta_{0 M}, \beta_{U L} \rightarrow 0\right)$ in the limit of the strong microfield $(A \rightarrow \infty)$. Therefore, we conclude that the suppression of the transition rates is due to a mixing of the forbidden and allowed transitions as a result of the Stark mixing. Supression of the transitions between $n=2$ sublevels due to Stark effect was reported earlier by Perrot ${ }^{11}$ through calculations of the inelastic collision cross sections in dense neon plasmas within the Born approximation.

The results shown in Figs. 2 and 3 may contain an error at high densities due to our use of the decoupling approximation (15) and quasistatic approximation (20b) for the above-mentioned ion-related term. It should be noted, however, that the $S_{\mathrm{ii}}^{(0)}$-related term in Eq. (24) is negligible for quadrupole transitions. ${ }^{3}$ Since substantial mixing of dipole and quadrupole transitions occurs through the Stark effect, the relative importance of the dipole transition is reduced and the resultant transition rates may not be so sensitive to the ion-related term.

We also note in Fig. 3 that in the low-density limit $(A$ $\rightarrow 0$ ), the four transition rates are nearly the same, because the level shifts are negligibly small and the strengths of the allowed transition are the same, i.e., $\beta_{U M}=\beta_{U L}=\beta_{0 M}$ $=\beta_{0 L}$.

In the numerical results shown in Figs. 1-3, the Stark effect was calculated at a particular field strength, $\mathcal{E}=\mathcal{E}_{0}$. To be more precise, we have to compute the transition rates for various values of $\mathcal{E}$ and to take an average over $\mathcal{E}$ with the aid of the distribution function $P(\mathcal{E})$ defined in Eq. (28). Perrot ${ }^{11}$ carried out such procedure for dense neon plasmas at $n_{e}$ $=1.09 \times 10^{24} \mathrm{~cm}^{-3}$ by using the Monte Carlo data of $P(\mathcal{E})$ for one-component plasmas. The resultant values of the excitation rates were close to those computed at $\mathcal{E}=\mathcal{E}_{0}$.

To evaluate $P(\mathcal{E})$, it is necessary to estimate the dielectric response function based on the arguments following Eqs. (26). For the numerical examples treated above, we find that the unscreened condition, Eq. (26a), is satisfied for $n_{e} \ll 1.1$ $\times 10^{24} \mathrm{~cm}^{-3}$, which covers the entire density range exhibited in Figs. 1-3. The electron-screening regime, Eq. (26b), is predicted for higher densities such as $1.1 \times 10^{24}$ $\ll n_{e}\left(\mathrm{~cm}^{-3}\right) \ll 2.0 \times 10^{27}$.

\section{CONCLUDING REMARKS}

The collisional excitation rates of an ion immersed in dense electron-ion TCP have been formulated through the time evolution equations of density matrices in a potential produced by the dynamical motions of plasma particles. The effect of energy level shifts in the target ion has been taken into consideration. Both the transition matrix elements and energy level shifts depend explicitly on the plasma density fluctuation operators. By adopting the RPA, these operators have been expressed in terms of the coordinates and velocities of the noninteracting dressed particles, where the cross term accounting for the electron-ion coupling has been properly retained.

Specifically, we have studied the electron-induced excitation, for which rapidly varying electron-density fluctuations have been decoupled from slowly varying ion-density fluctuations through comparison of the respective time scales. As the simplest approximation, the electron-impact line-broadening effect has been neglected, while the energy level shift due to the electric microfield produced by the screened ions has been properly taken into account within the quasistatic approximation. For a concrete example, we have computed the excitation rates among the $n=2$ finestructure levels of a hydrogen-like neon ion immersed in hydrogen plasmas. It has been demonstrated that excitation rates are considerably suppressed when the electric microfield is taken into account, because the allowed and forbidden transitions are mixed through the Stark effect. It has been shown that the energy level shift arising from the average polarization of the plasma plays a minor role.

Since the fine-structure levels are closely spaced, the transition rates are influenced by the slow electrons co- 
moving with the ions when the plasma density becomes sufficiently high. For these ion-related contributions, it would be inappropriate to treat the corresponding transitions and energy level shifts separately, and a dynamical description of the microfield would also be required. To achieve these ends, direct evaluation of Eq. (8) would be desirable. Finally, since the line shift and broadening are central issues in plasma spectroscopy, ${ }^{18}$ progress in that field would be beneficial to deeper understandings of the interplay between collisional excitation and energy level shifts in dense plasmas.

\section{ACKNOWLEDGMENTS}

The author thanks Dr. M. S. Murillo and Dr. J. C. Weisheit for pertinent discussions on this and related subjects.

\section{APPENDIX: DERIVATION OF EQS. (36)}

In order to evaluate the generalized oscillator strengths (19), we make use of the relation ${ }^{20}$

$$
\begin{aligned}
& \left\langle n l m|\exp (i \mathbf{k} \cdot \mathbf{r})| n^{\prime} l^{\prime} m^{\prime}\right\rangle \\
& =\sqrt{4 \pi} \sum_{L=0}^{\infty} \sum_{M=-L}^{L} i^{L} Y_{L M}^{*}(\hat{\mathbf{k}}) \text { ‘ } \\
& \times \int_{0}^{\infty} d r P_{n l}^{*}(r) j_{L}(k r) P_{n^{\prime} l^{\prime}}(r) \\
& \times(-1)^{m} \sqrt{(2 L+1)(2 l+1)\left(2 l^{\prime}+1\right)} \\
& \times\left(\begin{array}{lll}
l & L & l^{\prime} \\
0 & 0 & 0
\end{array}\right)\left(\begin{array}{ccc}
l & L & l^{\prime} \\
-m & M & m^{\prime}
\end{array}\right) .
\end{aligned}
$$

Here $P_{n l}(r)$ is the radial part of the wave function. For $n$ $=2$, we have

$$
\begin{aligned}
& P_{20}(r)=\frac{1}{\sqrt{2} a_{0}^{1 / 2}} \frac{r}{a_{0}}\left(1-\frac{r}{2 a_{0}}\right) \exp \left(-\frac{r}{2 a_{0}}\right), \\
& P_{21}(r)=\frac{1}{2 \sqrt{6} a_{0}^{1 / 2}}\left(\frac{r}{a_{0}}\right)^{2} \exp \left(-\frac{r}{2 a_{0}}\right),
\end{aligned}
$$

with $a_{0}=a_{\mathrm{B}} / Z$. The function

$$
\begin{aligned}
Y_{L M}(\hat{\mathbf{k}}) \equiv & (-1)^{(M+|M|) / 2} \sqrt{\frac{2 L+1}{4 \pi} \frac{(L-|M|)}{(L+|M|)}} \\
& \times P_{L}^{|M|}(\cos \theta) \exp (i M \varphi),
\end{aligned}
$$

refers to the spherical harmonics. The last two factors on the right-hand side of Eq. (A1) designate Wigner's 3-j symbols. ${ }^{20}$ The quantities $j_{L}(k r)$ and $P_{L}^{M}(\cos \theta)$ denote the spherical Bessel function and the associated Legendre function, respectively.

Though infinite summations over $L$ and $M$ are involved in Eq. (A1), only the terms satisfying the following three conditions contribute to the sum: $L+l+l^{\prime}=$ even, $\left|l-l^{\prime}\right|$ $\leqslant L \leqslant\left|l+l^{\prime}\right|$, and $M=m-m^{\prime}$. The transition matrix elements can thus be calculated as

$\langle 211|\exp (i \mathbf{k} \cdot \mathbf{r})| 200\rangle=\frac{3 i}{\sqrt{2}} \sin \theta \exp (-i \varphi) K \frac{1-K^{2}}{\left(1+K^{2}\right)^{4}}$,

$\langle 210|\exp (i \mathbf{k} \cdot \mathbf{r})| 200\rangle=-3 i(\cos \theta) K \frac{1-K^{2}}{\left(1+K^{2}\right)^{4}}$,

$\langle 211|\exp (i \mathbf{k} \cdot \mathbf{r})| 211\rangle$

$$
=\frac{1-K^{2}}{\left(1+K^{2}\right)^{4}}-\left(1-3 \cos ^{2} \theta\right) \frac{K^{2}}{\left(1+K^{2}\right)^{4}},
$$

$\langle 210|\exp (i \mathbf{k} \cdot \mathbf{r})| 210\rangle$

$$
\begin{gathered}
=\frac{1-K^{2}}{\left(1+K^{2}\right)^{4}}+2\left(1-3 \cos ^{2} \theta\right) \frac{K^{2}}{\left(1+K^{2}\right)^{4},} \\
\langle 200|\exp (i \mathbf{k} \cdot \mathbf{r})| 200\rangle=\frac{1-K^{2}}{\left(1+K^{2}\right)^{4}}-2 \frac{K^{2}}{\left(1+K^{2}\right)^{4}},
\end{gathered}
$$

with $K=k a_{0}$. Equations (A4) in conjunction with Eqs. (31) and (34) produce Eqs. (36).

${ }^{1}$ H. Kitamura, M. S. Murillo, and J. C. Weisheit, Phys. Plasmas 7, 3441 (2000).

${ }^{2}$ A. V. Vinogradov and V. P. Shevel'ko, Sov. Phys. JETP 4, 542 (1976).

${ }^{3}$ J. C. Weisheit, Adv. At. Mol. Phys. 25, 101 (1988).

${ }^{4}$ Th. Bornath, M. Schlanges, F. Morales, and R. Prenzel, J. Quant. Spectrosc. Radiat. Transf. 58, 501 (1997).

${ }^{5}$ M. S. Murillo and J. C. Weisheit, Phys. Rep. 302, 1 (1998).

${ }^{6}$ M. Inokuti, Rev. Mod. Phys. 43, 297 (1971).

${ }^{7}$ S. Ichimaru, Statistical Plasma Physics (Addison-Wesley, Reading, MA, 1992), Vol. I, Chaps. 1, 4, and 7.

${ }^{8}$ F. J. Rogers, H. C. Graboske, and D. J. Harwood, Phys. Rev. A 1, 1577 (1970).

${ }^{9}$ X.-Z. Yan and S. Ichimaru, Phys. Rev. A 34, 2173 (1986).

${ }^{10}$ H. Nguyen, M. Koenig, D. Benredjem, M. Caby, and G. Coulaud, Phys. Rev. A 33, 1279 (1986).

${ }^{11}$ F. Perrot, Phys. Scr. 37, 351 (1988).

${ }^{12}$ X.-Z. Yan and S. Ichimaru, Phys. Rev. A 34, 2167 (1986).

${ }^{13}$ M. S. Murillo, in Strongly Coupled Coulomb Systems, edited by G. J. Kalman, J. M. Rommel, and K. Blagoev (Plenum, New York, 1998), p. 607.

${ }^{14}$ E. L. Pollock and J. C. Weisheit, in Spectral Line Shapes, edited by F. Rostas (Gruyter, Berlin, 1985), Vol. 3, p. 181.

${ }^{15} \mathrm{H}$. A. Bethe and E. E. Salpeter, Quantum Mechanics of One- and TwoElectron Atoms (Plenum, New York, 1977), Sec. 55.

${ }^{16}$ N. Rostoker, Nucl. Fusion 1, 101 (1961).

${ }^{17}$ N. H. March and M. P. Tosi, Atomic Dynamics in Liquids (Dover, New York, 1976), Chap. 3.

${ }^{18}$ H. R. Griem, Principles of Plasma Spectroscopy (Cambridge University Press, Cambridge, U.K., 1997), Chap. 4.

${ }^{19}$ G. Lüders, Ann. Phys. (Leipzig) 8, 301 (1951).

${ }^{20}$ L. D. Landau and E. M. Lifshitz, in Quantum Mechanics, 3rd ed. translated by J. B. Sykes and J. S. Bell (Pergamon, Oxford, U.K., 1977), Secs. 39, 106, and 107.

${ }^{21}$ J. Lawson, W. Lawson, and M. J. Seaton, Proc. Phys. Soc. London 77, 192 (1961). 